\title{
High Resolution X-ray Imaging of a Globular Cluster Core: Compact Binaries in 47Tuc
}

\author{
Jonathan E. Grindlay', Craig Heinke, Peter D. Edmonds, and \\ Stephen S. Murray
}

\author{
Harvard-Smithsonian Center for Astrophysics, 60 Garden Street, \\ Cambridge, MA 02138
}

We have obtained high resolution $\left(\leqslant 1^{\prime \prime}\right)$ deep x-ray images of the globular cluster 47Tucanae (NGC 104) with the Chandra X-ray Observatory to study the population of compact binaries in the high stellar density core. A 70 kilosec exposure of the cluster reveals a centrally concentrated population of faint $\left(\mathrm{L}_{x} \sim 10^{30-33} \mathrm{erg} \mathrm{s}^{-1}\right)$ x-ray sources, with at least 108 located within the central $2^{\prime} \times 2.5^{\prime}$ and $\gtrsim$ half with $\mathrm{L}_{x} \lesssim 10^{30.5} \mathrm{erg} \mathrm{s}^{-1}$. All 15 millisecond pulsars (MSPs) recently located precisely by radio observations are identified, though two are unresolved by Chandra. The x-ray spectral and temporal characteristics, as well as initial optical identifications with the Hubble Space Telescope, suggest that $\gtrsim 50 \%$ are millisecond pulsars, $\sim 30 \%$ are accreting white dwarfs, $\sim 15 \%$ are main sequence binaries in flare outbursts and only 2 to 3 are quiescent low mass x-ray binaries containing neutron stars, the conventional progenitors of MSPs. An approximate upper limit of $\sim 470 M_{\odot}$ for the mass of an accreting central black hole in the cluster is derived. These observations provide the first x-ray "color-magnitude" diagram for a globular cluster and census of its compact object and binary population.

As the oldest stellar systems in the Milky Way Galaxy, globular clusters are laboratories for studies of stellar and dynamical evolution. The advantages of measurable ages and distances, both of which can be determined currently to $\sim 10 \%$, make globulars an especially attractive site for study of the evolution of stellar populations. The high stellar densities found in the cores of many globulars, with central values as high as $10^{6} M_{\odot} \mathrm{pc}^{-3}$ (1), implies that stellar interactions or near encounters can be relatively common - especially between stars and binary star systems. The role of binaries in cluster cores is dramatic: as

\footnotetext{
${ }^{1}$ To whom correspondence should be addressed. E-mail: josh@cfa.harvard.edu
} 
a source of localized gravitational binding energy, they act as a dynamical heat source in the cluster core, stabilizing it against further collapse into a central black hole (2).

Compact binary stars, in which one member is either a white dwarf (WD) or a neutron star (NS), are markers of the extremes of stellar and binary evolution. Together with short-period binaries containing main sequence stars, compact binaries interact with stars in globular cluster cores and affect the stellar populations directly. Stellar evolution within compact binaries containing either a WD or NS leads to mass transfer, and accretion onto the compact object makes them (and their progeny, MSPs) visible as persistent x-ray sources. X-ray flare emission, and fainter continuous emission, can also be detected from chromospherically active main sequence (M-S) binaries, known as BY Draconis systems. Thus x-ray observations are a particularly efficient way to study the compact binary population and the WD vs. NS population in globulars.

A complete sample of luminous x-ray sources $\left(\mathrm{L}_{x}(2-10 \mathrm{keV}) \gtrsim 10^{36} \mathrm{erg} \mathrm{s}^{-1}\right)$ has been discovered in globular clusters in the Galaxy: one bright source is detected (though nearly $50 \%$ are transient) in each of 12 clusters, most with high central stellar density and metallicity (3). Type I x-ray bursts (4) have been detected from each, indicating all are NSs accreting from lower mass binary companions. This was also suggested by the $\sim 3{ }^{\prime \prime}$-accuracy $\mathrm{x}$-ray positions measured for 6 of them with the Einstein x-ray Observatory which led to subsequent optical identifications for several and which yielded radial offsets consistent with their being $\sim 2 M_{\odot}$ objects (5). These 12 low mass x-ray binaries (LMXBs) in the system of $\sim 150$ globulars in the Galaxy are, as originally suspected (6), $\sim 200 \times$ more common (per unit mass) in globulars than in the Galaxy as a whole. Thus 2-body stellar (tidal) interactions between a NS and M-S star, and the more probable exchange interactions of a NS with a pre-existing binary of two M-S stars, enhances LMXB production in globulars. The likely descendants of LMXBs, MSPs, in which the NS has been spun up to millisecond spin periods by accretion and becomes visible as a radio pulsar after the accretion phase ends (7), are also favored in globulars. The relative LMXB vs. MSP populations in a globular can thus constrain the formation epoch of each and whether alternative production channels are required for MSPs, such as accretion induced collapse (AIC) of massive WDs (to NSs) which could directly produce MSPs without a progenitor LMXB phase (7). In the relatively massive and high central density cluster 47Tuc (NGC 104), at least 20 MSPs are now detected which imply a total population of $\sim 100-200$ in this cluster alone (8).

We obtained a 70 ksec exposure of 47Tuc on UT16.31 - 17.22 March, 2000, with the Chandra X-ray Observatory and Advanced CCD Imaging Spectrometer-Imager (ACIS-I) at its focus (9). Our primary goal was to study the population of low luminosity x-ray sources $\left(\mathrm{L}_{x} \lesssim 10^{33-34} \mathrm{erg} \mathrm{s}^{-1}\right)$ discovered in this and other globulars (10) and originally suggested 
to be primarily accreting WDs and thus cataclysmic variables $(\mathrm{CVs})$, together with a smaller population of the typically transient NS systems (LMXBs) in quiescence (quiescent LMXBs). Alternatively, the quiescent LMXB population may dominate the faint x-ray source population in globulars (11), and be required to maintain the large MSP population if they are derived continuously from LMXBs (7). Thus measurement and identification of a large sample of low luminosity x-ray sources in 47 Tuc, with its rich MSP population, can constrain the formation and evolution of MSPs as well as the relative populations of cluster WDs vs. NSs, with implications for the cluster initial mass function. We present results from a $2.0^{\prime} \times 2.5^{\prime}$ field centered on the cluster core and chosen to include all of the 15 MSPs with precise positions (12). This analysis for an inscribed radius of $1^{\prime}=\sim 2.6 \mathrm{r}_{c}$, where $\mathrm{r}_{c}$ is the cluster core radius (13), should include most compact binaries given their expected mass segregation (2). Results for the full $16^{\prime} \times 16^{\prime}$ ACIS-I field will be presented subsequently.

\section{Overview of Source Distributions}

Previous x-ray studies of 47Tuc with the ROSAT telescope and HRI detector (14) revealed a population of 9 sources with x-ray luminosity $\mathrm{L}_{x} \sim 3-100 \times 10^{31} \mathrm{erg} \mathrm{s}^{-1}$ within $\sim 1^{\prime}$ of the cluster center as well as underlying unresolved emission with total luminosity $\mathrm{L}_{x} \sim 4 \times 10^{32} \mathrm{erg} \mathrm{s}^{-1}$. The factor of $\gtrsim 10$ improvements in angular and spectral resolution (to $\sim 0.5^{\prime \prime}$ and $\sim 120 \mathrm{eV}$, respectively, with ACIS) achieved by Chandra over ROSAT enable detection of sources $\sim 10-100 \times$ fainter. The spectral resolution enables comparison with $\mathrm{x}$-ray spectra of known classes of source, while the exquisite positional resolution permits much more detailed searches for optical counterparts with high resolution HST images. Our deep exposure reached a sensitivity limit of $\mathrm{L}_{x} \sim 6 \times 10^{29} \mathrm{erg} \mathrm{s}^{-1}$ for a threshold source with 3 counts (vs. $\sim 0.5$ counts total background per source) detected at $90 \%$ confidence by the Chandra analysis tool wavdetect (15) and assuming a soft thermal bremsstrahlung spectrum with temperature $\mathrm{kT}=1 \mathrm{keV}$ (see below) for comparison with the ROSAT results (14). The "true" color image of the cluster core as imaged by Chandra (Fig. 1) shows the range of source brightness and spectral color by combining counts detected in soft $(0.5-1.2 \mathrm{keV}$; red), intermediate (1.2 - $2 \mathrm{keV}$; green) and hard (2 - $6 \mathrm{keV}$; blue) bands. Wavdetect finds 103 sources with total counts (in a $0.5-4.5 \mathrm{keV}$ primary detection band) from 3 (detection threshold) to 5332 over the total $70 \mathrm{ksec}$ exposure. Five additional sources are found at the positions of MSPs Q, L, T, M and C: Q, L, and T were missed by wavdetect due to crowding, while $\mathrm{M}$ and $\mathrm{C}$ are only 3 and 1 count "detections", though precisely at the MSP positions, due to low exposure $(\sim 30 \%)$ on the detector gaps. Using the recently derived distance $(5.0 \mathrm{kpc})$ and absorption for $47 \mathrm{Tuc}(16)$, we derive the luminosity distribution for the sources (Fig. 2). The complete list of 108 source positions and counts detected in

several bands, as well as source identifications currently derived (some discussed below), are 
given in the Supplementary Table (available at Science On Line).

The x-ray luminosity function increases at low values, with more than half the sources with $\mathrm{L}_{x} \lesssim 3 \times 10^{30} \mathrm{erg} \mathrm{s}{ }^{-1}$. The 23 sources in the lowest $\mathrm{L}_{x}$ bin $\left(2.5-7.5\right.$ cts; $\mathrm{L}_{x} \sim 10^{30}$ erg $\mathrm{s}^{-1}$ ) of the linear distribution form an incomplete set. From visual examination of the image as well as a 500 count excess of total counts over detected source counts in the central core region $\left(\mathrm{r} \lesssim 1 \mathrm{r}_{c}\right)$, we estimate an additional $\gtrsim 100$ sources with $\mathrm{L}_{x} \lesssim 10^{30}$ erg $\mathrm{s}^{-1}$ are missed with wavdetect due to crowding. Both a statistics-limited overlapping source detection algorithm (17) and a deeper ACIS-S exposure (with better low energy sensitivity) are needed to constrain the number and types of these faintest core sources.

The sources appear to be of (at least) 4 types as marked with different symbols in Fig. 1 and discussed below. The distribution of source types can also be seen in the x-ray "color magnitude" diagram (Fig. 3), which is possible to derive for the first time for a significant population of x-ray sources in a single globular cluster.

\section{Source Identifications}

Our analysis region of the cluster core was chosen to include the 15 MSPs with known positions (12) because 10 MSPs (including one in the globular cluster M28) have been detected in x-rays (18) and detection of even some in 47Tuc would enable a study of a population of cluster MSPs and their relation to both quiescent LMXBs and CVs. All 14 of the resolvable MSPs are detected (MSPs G and I, possibly a bound triple, have only $0.12^{\prime \prime}$ separation (12)), with exposure-corrected counts ranging from 3-27 and thus

$\mathrm{L}_{x} \sim 10^{29.8-30.8} \mathrm{erg} \mathrm{s}^{-1}$. Source identifications are possible, since even the faintest (3ct; $\gtrsim 2 \sigma$ in wavdetect) Chandra sources have positions with $\sim 0.2^{\prime \prime}$ uncertainties $(1 \sigma)$ due to the $\sim 0.8^{\prime \prime}$ Chandra image diameter (near the telescope axis) and 0.5" ACIS pixel size. The precise $\left(\sim 0.001^{\prime \prime}\right)$ radio timing positions of the MSPs permit an astrometric solution for the Chandra source positions. Using 7 of the brighter or best resolved Chandra/MSP source candidates, the rms deviation between the Chandra and precise MSP positions is only $0.11^{\prime \prime}$. Given a similarly precise Chandra-HST astrometric solution from our optically identified sources (see below), this will permit optical searches for the MSP counterparts. Because the 14 resolved Chandra MSP candidates are all located near the center of the circles which mark the precise MSP positions (Fig. 1), the identifications are very likely. Although the probability that one of the estimated $\sim 100$ unresolved sources in the 23 " radius core is within $0.5^{\prime \prime}$ of any MSP position is 0.05 , this is applicable to only MSPs L and T (the only MSPs in the core not detected automatically with wavdetect, and thus with positional uncertainties $\sim 1$ pixel $=0.5^{\prime \prime}$ instead of wavdetect centroid values of typically $\left.\lesssim 0.2^{\prime \prime}\right)$. The ACIS time resolution (nominal 3.2sec) does not permit analysis for pulsations, and the limited counts do not permit spectral analysis other than the determination of hardness 
ratios. The relatively narrow x-ray luminosity range implies a steep x-ray luminosity function, with typical $\mathrm{L}_{x}$ similar to the field MSPs detected outside of clusters (18), though the predominantly "red" x-ray colors (see below) are in contrast to some of the field MSPs with hard spectral components and the one in $\mathrm{M} 28$ with $\mathrm{L}_{x} \sim 10^{32-33} \mathrm{erg} \mathrm{s}^{-1}(18)$. Full details of the 47 Tuc MSP x-ray source characteristics will be presented separately (19).

Three other classes of sources are suggested by their x-ray spectra and temporal characteristics (Fig. 1). X5 and X7 are probable quiescent LMXBs, 13 sources are candidate CVs, and 6 sources appear to be main sequence (M-S) binaries detected in flare outbursts due to enhanced chromospheric activity. Each class is considered in turn to then enable constraints on the much larger unidentified population.

$\mathrm{X} 5$ and $\mathrm{X} 7$ are detected in the $0.5-4.5 \mathrm{keV}$ medium-energy band with 4435 and 5332 counts (hereafter medcts) and have spectra dominated by soft blackbody components with $\mathrm{kT}=0.31$ and $0.29 \mathrm{keV}$, respectively. Their apparently constant black body luminosities $\left(\log \mathrm{L}_{x}=32.8\right.$; comparable to that detected with ROSAT (14)) and implied emission radii of only $\sim 0.8 \mathrm{~km}$ suggest they are quiescent LMXBs in which the x-ray emission is dominated by incandescence of the hot NS, slowly cooling from a previous accretion outburst phase. Using a hydrogen-atmosphere model, which modifies the opacity and temperature, as demonstrated with Chandra ACIS-S spectra for the field quiescent LMXB Cen X-4 (20), more realistic NS radii of $\sim 10 \mathrm{~km}$ are derived (21). Our detailed spectral analysis of X5 and X7 also reveal a power law component (photon index 2.6 - 3) similar to that found for Cen X-4 and indicative of either residual accretion or a "propeller" spin-down luminosity source, as well as a possible line emission component (modeled (21) as a collisionally excited Raymond-Smith plasma with $\mathrm{kT}=1 \mathrm{keV}$ ), indicative of a thin corona or perhaps wind from the system. The integrated fluxes for these two components are $\sim 0.3$ to 0.5 and $\sim 0.2$ of the black body component, respectively. Although a statistical test (Kolmogorov-Smirnov) on photon arrival times of X7 shows no significant variations in flux, X5 shows dramatic dips and possible eclipses, which may also be detected in the optical counterpart we have identified with HST (22). Both X5 and X7 are most probably quiescent LMXBs given their similar luminosities and spectra to those tabulated (23) for the 6 known NS-quiescent LMXB systems in the field. However, the hot gas and possible wind component may indicate that X5 and X7 are luminous newly-born MSPs ablating their companions and still optically thick to their radio emission, because they are not among the currently located MSP sample (12). The lack of positive flux increases (i.e. flares; vs. the negative dips seen in X5) and otherwise constant flux would support, but not require, the MSP interpretation.

The 13 candidate CVs (cf. Fig. 1) identified thus far in our HST identification program (22) have blue stellar counterparts indicative of accretion disks (though not all are yet 
determined to be variable). Apart from the quiescent LMXBs X5 and X7, all remaining ROSAT sources [X6, X9, X10, X11, X13, and X19; (14)] within our analysis region (X4 is just outside) are CV candidates. Spectra have been fit (21) for the brightest 8 sources (with medcts $=135-2402$ ) and most are best fit with thermal bremsstrahlung with temperatures $\mathrm{kT}=5-30 \mathrm{keV}$, typical of CVs (24). Soft black body components, as found for quiescent LMXBs, are generally not present. Three sources have unusually hard spectra: X6 is best fit with a thermal bremsstrahlung spectrum with $\mathrm{kT} \gtrsim 75 \mathrm{keV}$, and both W8 and W15 require similarly hard spectra but with internal self-absorption column densities of $\mathrm{NH}=1.4 \pm 0.4$ and $0.7 \pm 0.2 \times 10^{22} \mathrm{~cm}^{-2}$, respectively. Power law fits are equally acceptable for all three but with photon index $\alpha \sim 1.1 \pm 0.1$ (consistent with the slope of a thermal bremsstrahlung spectrum). Since such a flat power law index at low energies $(<6 \mathrm{keV})$ is unprecedented for MSPs (for which the limited spectral data (18) indicate $\alpha \sim 2-3$ ) or active galactic nuclei (AGN) with typical (25) $\alpha \sim 1.7$, a thermal bremsstrahlung model is more likely. The excess NH in sources W8 and W15 is evident from their "blue" colors in Fig. 1 (absorption of soft counts) and might suggest they are background AGN, probably Seyfert 2 galaxies, even though the number expected (from hard sources in Chandra deep surveys (26)) in this $2^{\prime} \times 2.5^{\prime}$ field and exposure is $\lesssim 0.1$. However, not only are their power law spectral fits too hard, but our identification (22) of both W8 and W15 with blue stars imply x-ray/optical flux ratios similar to values expected for CVs (e.g. $\log \left(\mathrm{F}_{x} / \mathrm{F}_{v}\right)=+0.1$ for W15). X-ray self-absorption is likely in high inclination CVs (27) and may also arise from the "accretion curtain" (27), or relatively cool inner disk near the magnetospheric-disk interface suspected to exist in magnetic CVs in which accretion onto the WD is channeled by the magnetic field. Magnetic CVs also typically have relatively high temperature thermal bremsstrahlung components, though the $\mathrm{kT}$ values for X6, in particular, is unusually large.

The brightest CV candidate, X9 (medcts $=2402$ ), is well fit with a thermal bremsstrahlung spectrum with $\mathrm{kT}=32 \pm 6 \mathrm{keV}$ and is coincident $\left(0.1^{\prime \prime}\right)$ with the blue $\mathrm{CV}$ candidate V1 (28), confirming the identification by the ROSAT detection (14) of X9. The $70 \mathrm{ksec}$ Chandra observation of X9 shows evidence for pulsations (false alarm probability $\sim 2 \times 10^{-3}$ ) with period $\mathrm{P}=218.24$ s and modulation depth $30 \%$ (Fig. 4), suggesting this is direct evidence for an accreting magnetic WD and thus magnetic CV. Although the total flux from X9 is relatively constant (Fig. 5), and a factor of $\sim 2$ fainter than detected with ROSAT, the pulsation amplitude is variable (strongest in the second quarter of the observation) and could instead indicate transient pulsations as found in some CVs (24). The faint CV candidate AK09 is also a possible magnetic CV. It was not detected with ROSAT and is too faint even with Chandra (medcts $=36$ ) for spectral fitting but is relatively soft (red; cf. Fig. 1). The Chandra position is coincident (0.1") with the blue variable AK09 (29) with $1.1 \mathrm{~d}$ binary period, and originally suggested (30) as the counterpart of the single 
x-ray source detected in the original Einstein x-ray image of 47 Tuc (5), for which the positional uncertainty ( $3 "$; 90\%) included both X9 and AK09. HST spectra (31) now show AK09 to be almost identical to the magnetic CV system GK Per, with similarly long (2d) binary period and occasional outbursts. AK09 also shows UV outbursts (32) so that the bright Einstein source, which had both a thermal bremsstrahlung spectrum (kT $=2-6 \pm 1$ $\mathrm{keV}$ ) as well as possible 120.2s pulsations (30), might have been AK09 in outburst. Both Chandra (21) and HST (22) studies further constrain the magnetic CV hypothesis.

The optically discovered CV, V2 (33), which displays dwarf nova-like outbursts is identified $\left(0.1^{\prime \prime}\right)$ with source X19 and fit with a thermal bremsstrahlung spectrum with $\mathrm{kT}=12 \pm 6 \mathrm{keV}$, and the blue variable V3 (32) is the probable counterpart (0.1") for $\mathrm{X} 10$, in contrast to the claim from ROSAT data (14). The Chandra spectrum of X10 is unique among all the (moderately) bright sources for which spectral fits were possible. X10 can be fit with a pure power law $(\alpha=2.7 \pm 0.2)$. Its x-ray color and extreme variability, with dips or flares and eclipses (Fig. 5), for which a power spectrum reveals a significant $3.83 \mathrm{~h}$ period, are similar to the quiescent LMXB candidate X5 although a black body + power law component (as for X5) is only acceptable if a Raymond-Smith component is added in absorption. However, its blue variable counterpart V3, with Balmer emission lines (34), suggests accretion is ongoing. We suggest X10, and thus perhaps X5, may be similar to the peculiar magnetic CV AE Aqr, in which spin energy of the WD is partly converted to non-thermal radiation by a magnetic propeller, possibly anchored in the disk (35). Alternatively, the power law spectrum suggests X10 might be, as mentioned for X5, an enshrouded MSP.

The fourth group of probable source identifications are main sequence binaries. Of the 6 sources marked (Fig. 1), the brightest, identified with the 12.7h binary E8 (29), is the most convincing association with what may be chromospherically active M-S binaries. No blue excess is found in our HST analysis (22), and the star appears just above the main sequence. This is as expected for a BY Dra star, the main sequence analog of RS CVn systems in which x-ray emission is produced primarily in giant flare outbursts. Although the $\mathrm{L}_{x}=10^{31.4} \mathrm{erg} \mathrm{s}^{-1}$ is a factor of $\sim 10$ larger than the maximum found with ROSAT (36) for field BY Dra systems in quiescence, this is probably due to what appears to be a smooth decline from a large outburst (Fig. 5). The other 5 candidate BY Dra systems (Fig. 1) also show x-ray flare-like variations. Using our Chandra-HST astrometry, we have found at least 10 other optical variables without blue excesses that are possible BY Dra systems, although without x-ray flare variations they are less likely identifications. Few, if any, RS CVn systems are seen, with only 2-3 possible matches $\left(\lesssim 0.5^{\prime \prime}\right)$ of Chandra sources with sub-giants. 


\section{Implications for Population of Compact Objects, Binaries and Cluster Core}

While source types for some individual cases are uncertain (e.g. X10), it appears that the 108 sources near the core of 47Tuc are a mixture of 4 source types. Their quasi-continuous distribution in x-ray colors (Fig. 3) is due to the relative insensitivity of broad-band colors to detailed spectral differences, particularly for multi-component spectra. Nevertheless, some broad differences emerge when the distribution of x-ray color is examined (Fig. 6). Most striking is that most of the MSPs (for which identifications are unambiguous) are relatively soft: 8 of the 12 with medcts $\gtrsim 5$ (and thus rough colors available, as in Fig. 3) are softer than any of the much brighter CV candidates. Similarly, the two most probable quiescent LMXBs are softer than all of the CVs (except AK09) and are intermediate between the CVs and MSPs. The BY Dra candidates (BYs) are intermediate in color but closer to the CVs. Although the statistics are limited, the values for the mean, $\overline{X \text { color }}$, and standard deviation, $\sigma_{\text {xcolor }}$, for each population are: $\overline{\text { Xcolor }}$, $\left.\sigma_{\text {xcolor }}\right)=(1.8 \pm 0.3,0.98),(-0.1 \pm 0.3,1.09),(0.8 \pm 0.2,0.56)$ and $(1.5 \pm 0.1,0.17)$ for MSPs, CVs, BYs, and quiescent LMXBs respectively. The corresponding distribution values for the unidentified (UnID) sources are $(1.1 \pm 0.1,1.03)$, or between the BYs and MSPs. The BY contribution to the UnIDs can be measured by a deep followup observation. Most of the same UnID sources will not be detected if they are flare outbursts from BY Dra systems, while MSPs will be constant in their x-ray emission. Given the approximate relative numbers of the identified sources and the Xcolor distributions (Fig. 6), we decompose the 61 UnID (>5ct)source distribution into 2 quiescent LMXBs, 18 CVs, 11 BYs, and 30 MSPs. Combined with the identified sources, this yields approximate relative (fractional) contributions of $0.04,0.3,0.16$ and 0.5 for the populations of quiescent LMXBs, CVs, BYs, and MSPs with $\mathrm{L}_{x} \gtrsim 10^{30} \mathrm{erg} \mathrm{s}^{-1}$, respectively, where the uncertainties are probably $30 \%$.

We conclude the source population in 47Tuc is dominated by MSPs, with a total number of $\gtrsim 45-60$ in the present Chandra sample (with $\mathrm{L}_{x} \gtrsim 1 \times 10^{30} \mathrm{erg} \mathrm{s}^{-1}$ ). These would naturally account for the background or unresolved red sources, suggesting a still larger MSP population at lower luminosities, if these faint sources are detected as constant flux sources in followup observations. Further inferences for the MSP population, including their spindown energy loss, $\dot{E}$, vs. $\mathrm{L}_{x}$ correlations and radial distributions in the cluster, are discussed separately (19). However, the CV population may also extend into this faint, soft source background population if there are large numbers of strongly magnetic (AM Her type) CVs for which soft black body emission dominates. These would be optically very faint (like the MSPs), but would display marked variability (relatively long high-low states), and would thus also be distinguished from both BY Dra and MSP systems of comparable $\mathrm{L}_{x}$. 
Our estimate that the present sample contains $\sim 30 \mathrm{CVs}$ is $\sim 1 / 3$ the number predicted for tidal capture CV production models (37). This may indicate tidal capture is relatively inefficient, but in fact the prediction of a large excess of low luminosity sources $\left(\mathrm{L}_{x} \lesssim 10^{30}\right.$ erg s$~^{-1}$ ) may also be consistent with our "background" sources. The size of the CV sample is unexpected because so few dwarf novae have been found (33). Combined with the evidence presented here that the brightest CV candidates (e.g. X9) are magnetic CVs, it may be that $\mathrm{CVs}$ in globulars are indeed dominated by magnetic systems, as suggested from HST spectra (38), in which dwarf nova outbursts are suppressed, at least for short binary period systems.

If only 2-3 quiescent LMXBs (X5, X7, and possibly X10) are present in the core with the large sample of $\sim 100$ MSPs inferred here or from radio counts (8), the spin-up timescales for MSPs are reduced to $\mathrm{T}_{\text {spinup }} \sim \mathrm{N}_{\text {quiescentLMXBs }} / \mathrm{N}_{M S P s} \times \mathrm{T}_{M S P s}$, where $\mathrm{T}_{M S P s} \sim 3 \times 10^{9}$ years is the typical MSP spindown age. Thus $\mathrm{T}_{\text {spinup }} \sim 1 \times 10^{8}$ years, which is $\sim 10 \times$ faster than the $\gtrsim 10^{9}$ years needed to transfer the $\sim 0.03 \mathrm{P}_{4}^{-4 / 3} M_{\odot}$ required $(7)$ to spin up the NSs in 47 Tuc to their typical $\sim 4 \mathrm{msec}$ spin period $\left(\mathrm{P}_{4}\right.$ is the final period in units of $\left.4 \mathrm{msec}\right)$ at the time-averaged mass accretion rate $\dot{m} \sim 3 \times 10^{-11} M_{\odot} \mathrm{yr}^{-1}$ appropriate to typical LMXBs in clusters with $\mathrm{L}_{x} \sim 10^{36.3} \mathrm{erg} \mathrm{s}^{-1}(3)$ which may be in quiescence $\sim 50 \%$ of the time. Thus the observed quiescent LMXBs are insufficient to support the MSP population and alternative paths to MSP production may be required such as direct collapse of massive WDs in high $\dot{m} \mathrm{CVs}$ (7) (for which X9 may be relevant) or MSP formation from common envelope evolution of a NS with a prior generation of $\sim 1-3 M_{\odot}$ binary companions leading to a short-lived NS-WD LMXB phase (39).

Finally, the Chandra image allows the most sensitive mass limits for a black hole in the core of a globular cluster. The precise MSP positions and associated pulsar dispersion measures (12) imply an electron density (and thus ionized gas at $\mathrm{T} \sim 10^{4} \mathrm{~K}$ ) of $\mathrm{n} \sim 0.1$ $\mathrm{cm}^{-3}$ in the cluster core. Bondi-Hoyle spherical accretion with radiative efficiency $\epsilon$ onto a compact object of mass $\mathrm{M}$, radius $\mathrm{R}$ and velocity $\mathrm{V}$ in a gas with number density $\mathrm{n}$ and sound speed $\mathrm{c}_{s}$ gives an accretion luminosity $\mathrm{L}_{a c c}=\epsilon \mathrm{G} \mathrm{M}^{3} \mathrm{~m}_{p} \mathrm{n}\left(\mathrm{V}^{2}+\mathrm{c}_{s}^{2}\right)^{-3 / 2} \mathrm{R}^{-1}$, where $\mathrm{m}_{p}$ is the proton mass. For an isolated NS as recently considered for low luminosity x-ray sources in globulars (40), this gives $\mathrm{L}_{a c c}=10^{30} \mathrm{erg} \mathrm{s}^{-1}$ for 47Tuc and appropriate NS values of $\mathrm{M}_{n s}=1.4 M_{\odot}, \mathrm{R}_{n s}=10 \mathrm{~km}, \epsilon=0.1$ and $\mathrm{V}_{n s} \sim \mathrm{c}_{s} \sim 10 \mathrm{~km} / \mathrm{s}$. If the accretion energy is thermalized over the NS surface, the expected black body spectrum with $\mathrm{kT} \sim 15 \mathrm{eV}$ is too soft to account for the detected Chandra sources. Although the thermalization process is uncertain, higher temperature radiation from a magnetic pole is also inhibited at such low accretion rates in the spherical case if the NS is rapidly rotating. However, for a black hole, with larger mass $\mathrm{M}_{b h}$, emission radius $\sim 3$ Schwarzshild radii and lower equipartition velocity $\mathrm{V}_{b h}=\mathrm{V}_{n s}\left(\mathrm{M}_{n s} / \mathrm{M}_{b h}\right)^{1 / 2}$ (so that $\mathrm{c}_{s}$ dominates), the accretion luminosity will 
likely arise from a much lower efficiency flow with $\epsilon \sim 10^{-4}$ and radiate a much higher temperature spectrum ( $\mathrm{kT} \sim 100 \mathrm{keV}$ ), such as calculated in advection (or convection) dominated accretion models (41) for accretion on the black hole x-ray source SgrA* in the galactic center. We estimate a soft x-ray luminosity $\mathrm{L}_{x}(0.5-2.5 \mathrm{keV}) \sim 4.5 \times 10^{25} \epsilon_{-4} \mathrm{M}_{b h}^{2} \mathrm{~T}_{100}$ $\operatorname{erg~\mathrm {s}^{-1}}$, where the uncertain radiation efficiency $\epsilon$ and spectral temperature $\mathrm{T}$ are scaled to $10^{-4}$ and $100 \mathrm{keV}$, respectively. The Chandra upper limit of $\mathrm{L}_{x} \sim 1 \times 10^{31} \mathrm{erg} \mathrm{s}^{-1}$ for the brightest source in the cluster center error circle (42) (Fig. 1) implies an upper limit of

$\mathrm{M}_{b h} \sim 470 M_{\odot} \epsilon_{-4}^{-1 / 2} \mathrm{~T}_{100}$. This is consistent with the understanding that binary heating $(2)$ prevents core collapse into a moderately massive central black hole and that stellar mass $\left(\sim 3-10 M_{\odot}\right)$ black holes produced from the initial stars more massive than the NS cutoff have not coalesced in the core but rather have been ejected by the hardest binaries (43). A more precise cluster center and accretion model can improve these limits for $\mathrm{M}_{b h}$, which are already below the $1700 M_{\odot}$ value derived from the central surface brightness profile (44). Similar limits for those post core collapse clusters which, like 47Tuc, do not contain a bright LMXB and for which central gas densities could be estimated from MSPs, would be especially interesting.

\section{References and Notes}

1. G. Meylan and C. Pryor, in Dynamics of Globular Clusters, S. Djorgovski and G. Meylan, eds., ASP Conf. Series, 50, 31 (1993).

2. P. Hut et al., Publ. Astron. Soc. Pacific, 104, 981 (1992).

3. J. E. Grindlay, The Globular Cluster Galaxy Connection, G.H. Smith and J.P. Brodie, eds, ASP Conf. Ser., 48, 156 (1993); and L. Sidoli et al, Astron. Astrophys., in press (and available at http://xxx.lanl.gov/abs/astro-ph/0012383).

4. W. Lewin, J. van Paradijs and R. Taam, in X-ray Binaries, W. Lewin, J. van Paradijs and E. van den Heuvel, eds. (Cambridge Univ. Press: Cambridge, 1995), 175.

5. Precise $\left(\lesssim 3^{\prime \prime}\right)$ x-ray positions for globular cluster LMXBs as measured by J.E. Grindlay, P. Hertz, J. Steiner, S. Murray, and A. Lightman Astrophys. J., 282, L13 (1984) enabled mass estimates for the sources and subsequent optical identifications for several as described by L. Homer, S. Anderson, B. Margon and E. Deutsch, Astrophys. J., 550, L155 (2001) and references therein.

6. G. Clark Astrophys. J., 199, L143 (1975) and J. Katz, Nature, 253, 698 (1975). 
7. D. Bhattacharya and E.P. van den Heuvel, Physics Reports, 203, 1 (1991) provide a comprehensive review of LMXB and MSP evolution. The AIC model for production of MSPs directly from collapse of WDs was proposed to solve the possible LMXB vs. MSP birthrate problem in globulars by J. Grindlay and C. Bailyn, Nature, 336, 48 (1988).

8. F. Camilo, D.R. Lorimer, P. Freire, A.G. Lyne, and R.N. Manchester, Astrophys. J., 535, 975 (2000).

9. The Chandra X-ray Observatory is described by M. Weisskopf, S. O'Dell and L. van Speybroeck, Proc. SPIE, 2805, 142 (1996); the ACIS detector system is described by G. Garmire, J. Nousek and M. Bautz, in preparation.

10. P. Hertz and J. Grindlay, Astrophys. J.275, 105 (1983).

11. F. Verbunt, R. Elson and J. van Paradijs, Mon. Not. R. Astron. Soc., 210, 899 (1984)

12. P.C. Freire, F. Camilo, D.R. Lorimer, A.G. Lyne, R.N. Manchester, and N. D'Amico, Mon. Not. R. Astron. Soc., in press (2001) (and available at http://xxx.lanl.gov/abs/astro-ph/0103372).

13. The core radius, $\mathrm{r}_{c}$, is defined as the radius at which the projected surface brightness has decreased by a factor of 2 from its peak central value. It is a measure of central stellar density and can be related to cluster dynamical parameters (see ref. (1)). The most reliable determination of $\mathrm{r}_{c}=23^{\prime \prime}$ for 47Tuc is given by J.H. Howell, P. Guhathakurta, and R.L. Gilliland, Publ. Astron. Soc. Pacific, 112, 1200 (2000).

14. F. Verbunt and G. Hasinger, Astron. Astrophys., 336, 895 (1998) report the results of the x-ray survey of 47Tuc carried out with the German-US x-ray telescope ROSAT (Rontgen Satellit) and its High Resolution Imager (HRI) detector.

15. Wavdetect is a source detection tool employing wavelet smoothing to match the expected point spread function. Along with the spectral analysis package, Sherpa, used for spectral fits to the brighter ( $\gtrsim 300 \mathrm{ct}$ ) sources, it is part of the Chandra Interactive Analysis Operation (CIAO) developed by the Chandra X-ray Center (CXC) for analysis of Chandra data and available at http://asc.harvard.edu/ciao/.

16. M. Zoccali, A. Renzini, S. Ortolani, A. Bragaglia, R. Bohlin, E. Carretta, F. Ferraro, R. Gilmozzi, J. Holberg, G. Marconi, R. Rich, F. Wesemael, preprint (2001) (and available at http://xxx.lanl.gov/abs/astro-ph/0101485). 
17. A 2D KS-test method to detect overlapping but statistics-limited sources has been developed by S. Metchev and J. Grindlay Astrophys. J., in preparation (2001); maximum likelihood methods are used in ref. (14).

18. W. Becker and J. Trumper, Astron. Astrophys., 341, 803 (1999).

19. J. Grindlay, F. Camilo, P. Edmonds and C. Heinke, in preparation (2001).

20. R.E. Rutledge, L. Bildsten, E.F. Brown, G.G. Pavlov, and V. E. Zavlin, Astrophys. $J$. , in press (2001) (and available at http://xxx.lanl.gov/abs/astro-ph/0012400).

21. C. Heinke, J. Grindlay and P. Edmonds, in preparation (2001). Spectral models fit to the cluster sources included both continuum (black body, bremsstrahlung and power law) and emission line (Raymond-Smith collisional excitation) models, as provided in the CIAO analysis package (see ref. 15).

22. P. Edmonds, R. Gilliland, J. Grindlay, C. Heinke et al, in preparation (2001).

23. M. Garcia, J. McClintock, R. Narayan, P. Callanan and S. Murray, Astrophys. J., in press (2001) (and available at http://xxx.lanl.gov/abs/astro-ph/0012452).

24. B. Warner, Cataclysmic Variables, (Cambridge Univ. Press: Cambridge, 1995).

25. A. Zdjiarski in ASP Conf. Series, 161, 16 (1999) provides a recent review.

26. R. Giaconni, P. Rosati, P. Tozzi, M. Nonino, G. Hasinger, J. Bergeron, S. Borgani, R. Gilli, R. Gilmozzi and W. Zheng, preprint (and available at http://xxx.lanl.gov/abs/astro-ph/0007240).

27. A. van Teesling, K. Beurmann and F. Verbunt, Astron. Astrophys., 315, 467 (1996) suggest absorption by the disk in high inclination non-magnetic CVs, whereas accretion curtain absorption in magnetic systems is proposed by C. Hellier, in Annapolis Workshop on Magnetic Cataclysmic Variables, C. Hellier and K. Mukai, eds., ASP Conf. Ser., 157, 1 (1999).

28. F. Paresce, G. de Marchi and F. Ferraro, Nature, 360, 46 (1992).

29. P. Edmonds, R. Gilliland, P.. Guhathakurta, L. Petro, A. Saha, and M. Shara, Astrophys. J., 468, 241 (1996).

30. M. Auriere, L. Koch and S. Ortolani, Astron. Astrophys., 214, 113 (1989).

31. C. Knigge, M. Shara, D. Zurek, K. Long and R. Gilliland, preprint (and available at http://xxx.lanl.gov/abs/astro-ph/0012187). 
32. D. Minniti, G. Meylan, C. Pryor, E. Phinney, B. Sams, and C. Tinney, Astrophys. J., 474, L27 (1997).

33. M. Shara, L. Bergeron, R. Gilliland, A. Saha and R. Petro, Astrophys. J., 471, 804 (1996).

34. P. Edmonds et al in preparation (2001).

35. K. Horne, in Annapolis Workshop on Magnetic Cataclysmic Variables, C. Hellier and K. Mukai, eds., ASP Conf. Ser., 157, 349 (1999).

36. R. Dempsey, J. Linsky, T. Fleming and J. Schmitt, Astrophys. J., 478, 358 (1997).

37. R. Di Stefano and S. Rappaport, Astrophys. J., 423, 274 (1994).

38. J. Grindlay, in Annapolis Workshop on Magnetic Cataclysmic Variables, C. Hellier and K. Mukai, eds., ASP Conf. Ser., 157, 377 (1999); and P. Edmonds, J. Grindlay, A. Cool, H. Cohn, P. Lugger and C. Bailyn, Astrophys. J., 516, 250 (1999).

39. F. A. Rasio, E.D. Phahl and S. Rappaport, Astrophys. J., 532, L47 (2000).

40. E. Pfahl and S. Rappaport, Astrophys. J., in press (2001) (and available at http://xxx.lanl.gov/abs/astro-ph/0009212).

41. E. Quataert and R. Narayan, Astrophys. J., 520, 298 (1999).

42. D. Calzetti, G. DeMarchi, F. Paresce, and M. Shara, Astrophys. J., 402, L1 (1993); and G. De Marchi, F. Paresce, M. Stratta, R. Gilliland, R. and Bohlin, Astrophys. J., 468, L51 (1996).

43. S.F. Portegies Zwart and S. McMillan, Astrophys. J., 528, L17 (2000).

44. P. Guhathakurta, B. Yanny, D. Schneider and J. Bahcall, in Dynamics of Globular Clusters, S. Djorgovski and G. Meylan, eds., ASP Conf. Series, 50, 303 (1993).

45. We thank F. Camilo, H. Cohn and R. Gilliland for helpful comments. This observation was conducted as part of the GTO program on the Chandra X-ray Observatory with support from NASA (HRC contract NAS8-38248). 

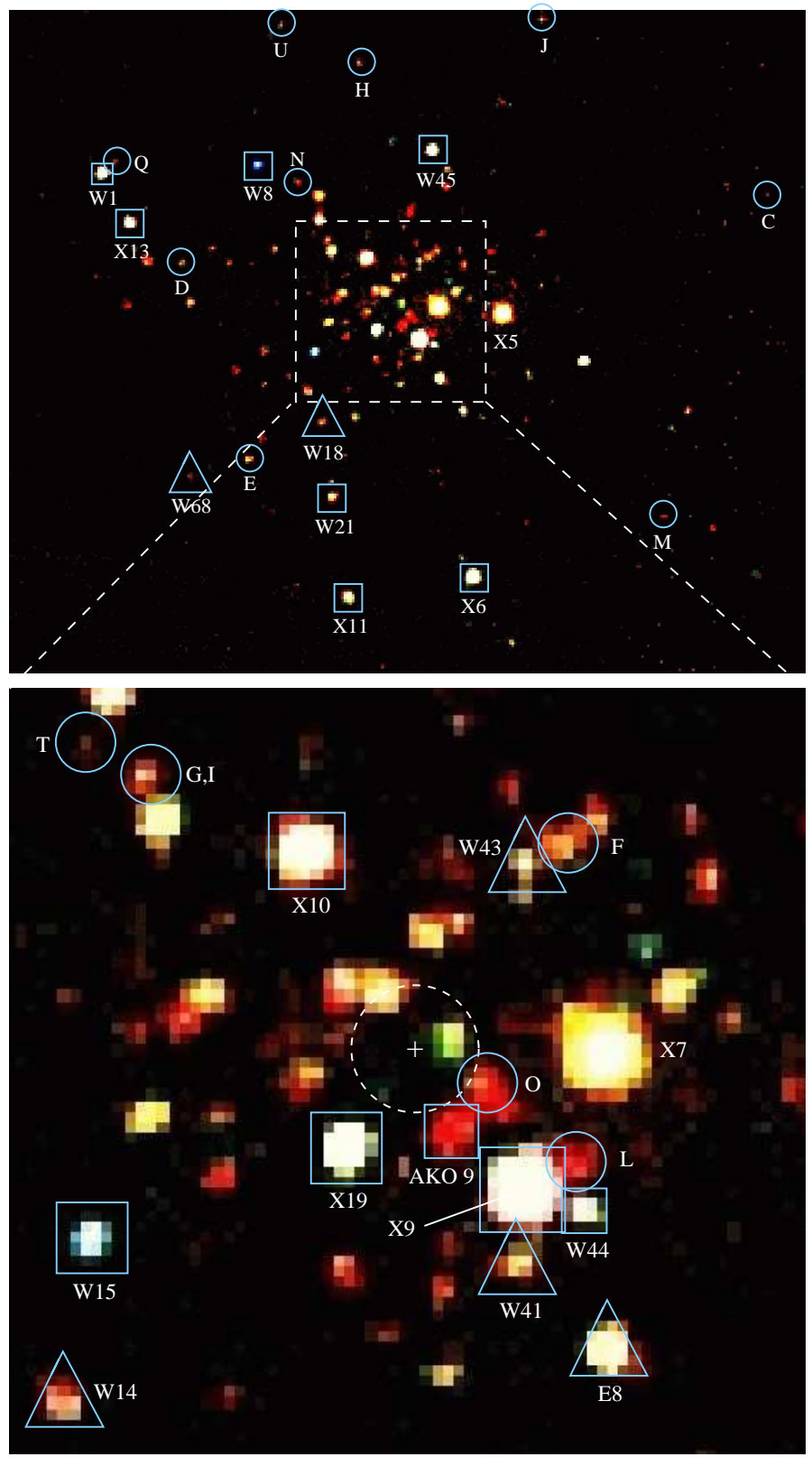

Fig. 1. X-ray color image of the central $2^{\prime} \times 2.5^{\prime}$ of 47 Tuc. Colors are derived from counts detected in soft (0.5 - $1.2 \mathrm{keV}$; red), intermediate (1.2 - $2 \mathrm{keV}$; green) and hard (2 - $6 \mathrm{keV}$; blue) bands with the I3 chip of the ACIS-I detector on Chandra. The pixel size (visible in enlargement of central core) is 0.4914 ", and the enlarged central region is $35^{\prime \prime}$ square. Source identifications are: MSPs (circles), marked with source letter names (12); quiescent LMXBs X5 and X7; CV candidates (squares), marked with ROSAT Xnumbers (14) or other Chandra Wnumbers; and possible flaring BY Dra systems, or M-S binaries (triangles), marked with E8 (29) or Chandra Wavdetect source numbers (Wnumbers). Precise positions, fluxes, hardness ratios, bright source spectra and variability are tabulated for all 108 sources shown here $(73$ unidentified are not labeled, for clarity) in our full-field analysis (21), with only key values given here in the text. The mean cluster center (41) and $3 \sigma$ error ( 3 " radius dashed circle) are marked; other symbols (squares, etc.) are centered on sources but with sizes much greater than positional uncertainties. 

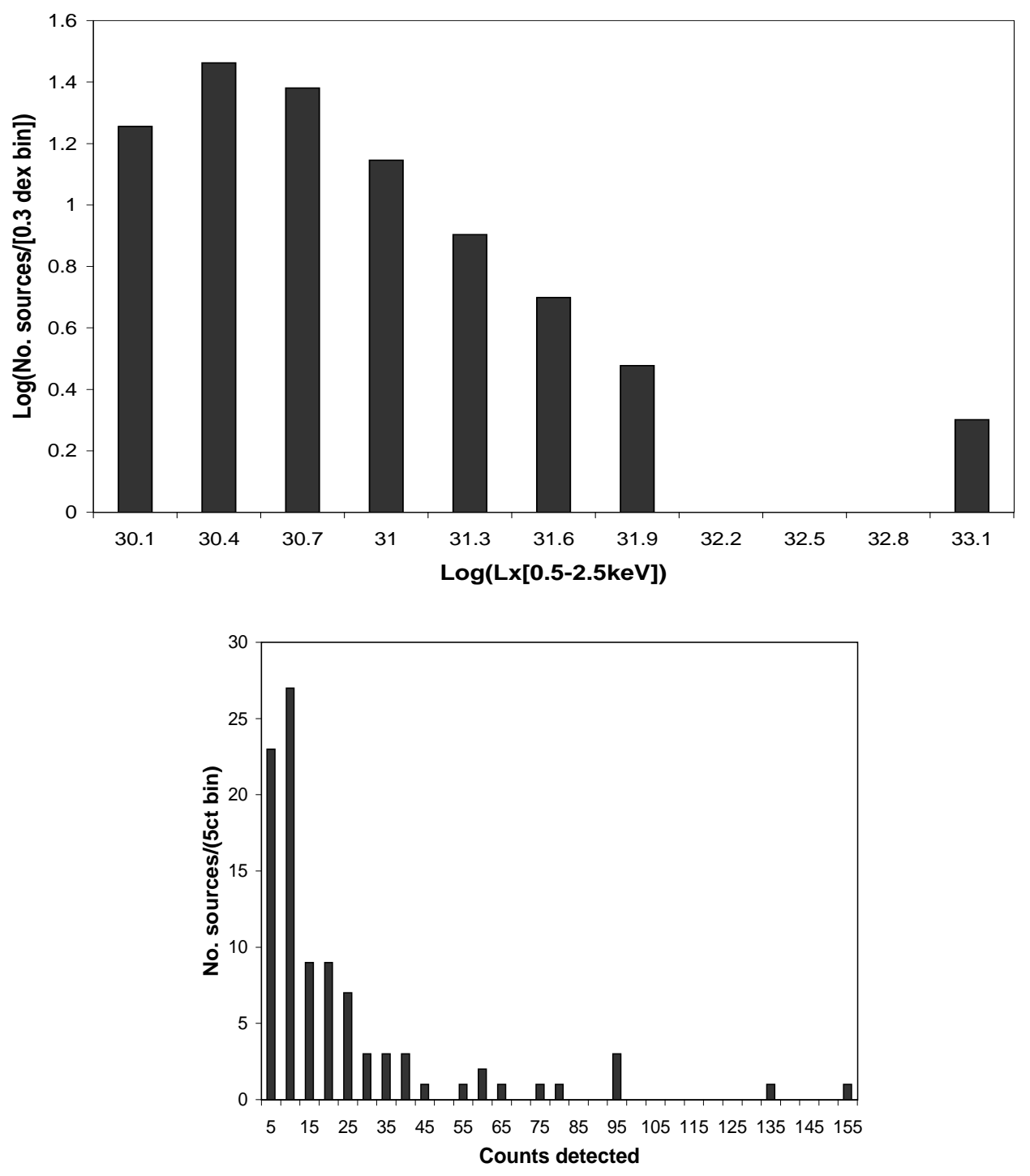

Fig. 2. X-ray luminosity and counts distributions of sources detected with $>2.5$ cts in central $2^{\prime} \times 2.5^{\prime}$ of 47 Tuc. Counts detected in medium band $(0.5-4.5 \mathrm{keV})$ are corrected for instrument and telescope response, $5.0 \mathrm{kpc}$ cluster distance (16) and known interstellar absorption of $\mathrm{A}_{V}=0.12$ (or equivalent column density $\mathrm{NH}=2.4 \times 10^{20} \mathrm{~cm}^{-2}$ ) and an assumed thermal bremsstrahlung spectrum with $\mathrm{kT}=1 \mathrm{keV}$ to give source luminosity in the $0.5-2.5 \mathrm{keV}$ band for direct comparison with ROSAT results (14). The extended energy band of Chandra $(0.5-8 \mathrm{keV})$ is utilized for hardness ratio and spectral analysis, which also enable conversion of $\mathrm{L}_{x}$ between the Chandra and ROSAT bands. (A) $\log$ source counts vs. $\log \mathrm{L}_{x}$, with $\log \mathrm{L}_{x}$ value given at the center of 0.3 dex bins, (B) linear source counts vs. actual counts detected, with value given at center of 5 ct wide bins. Corresponding $\log \mathrm{L}_{x}$ values range from 29.9 to 31.4. The 96 sources plotted in (B) account for only $13 \%$ of the total detected x-ray flux from the cluster central region, whereas the brightest two sources (probable quiescent LMXBs X5 and X7) contribute $57 \%$ of the total counts. 


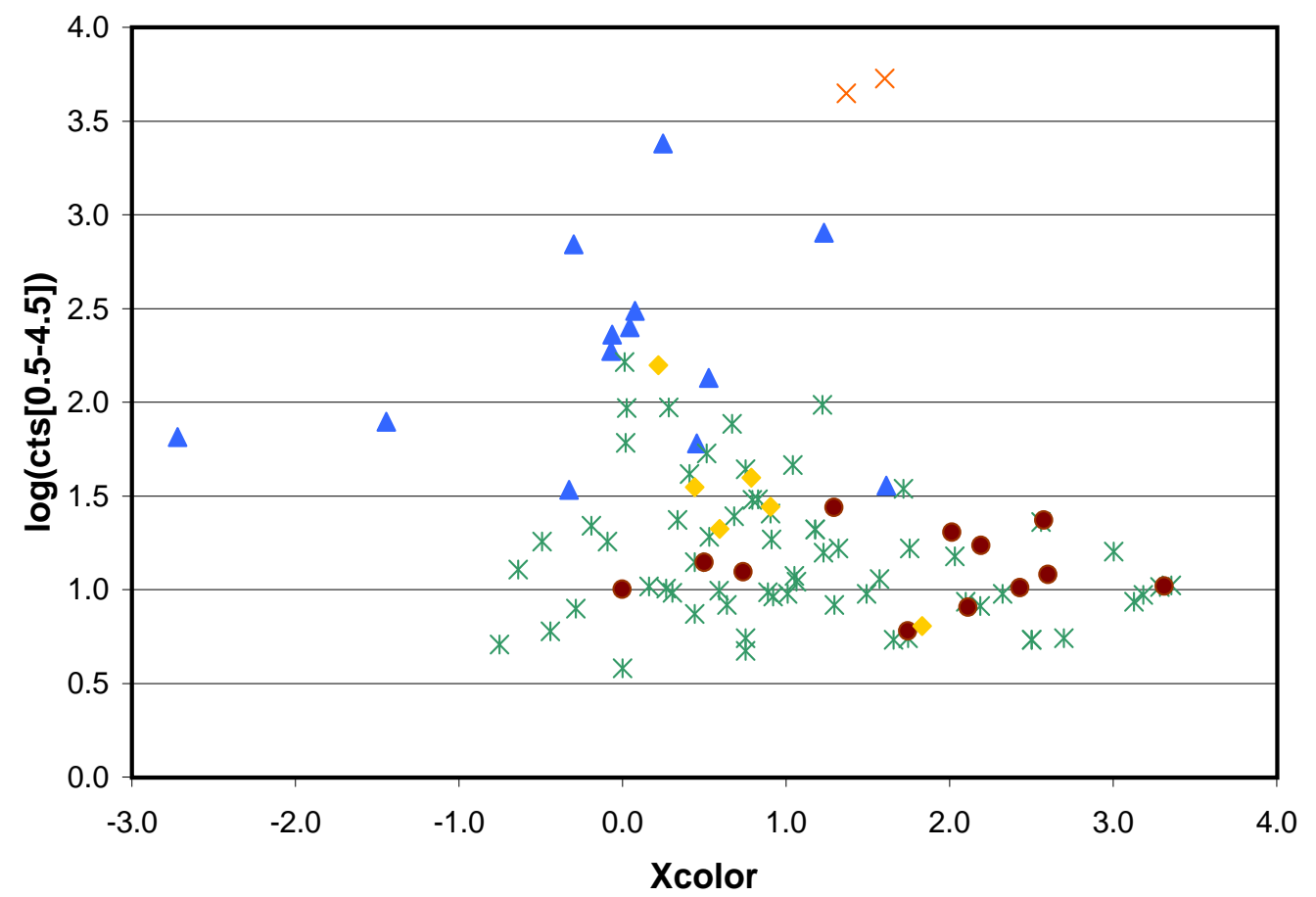

Fig. 3. X-ray brightness (medcts $=0.5-4.5 \mathrm{keV})$ vs. color (ratio of $\operatorname{soft}(0.5-1.5 \mathrm{keV}) / \mathrm{hard}(1.5-6 \mathrm{keV})$ counts) distribution for sources with medcts $\gtrsim 5$ cts. Source types are labeled for the sources identified with 12 MSPs (red •), 2 quiescent LMXBs (orange X), 13 CVs (blue $\triangle$ ) and 6 M-S binaries (BY Dra systems) in flare outbursts (yellow $\diamond)$. The 61 remaining (green $*$ ) are unidentified. Source hardness increases to left. 


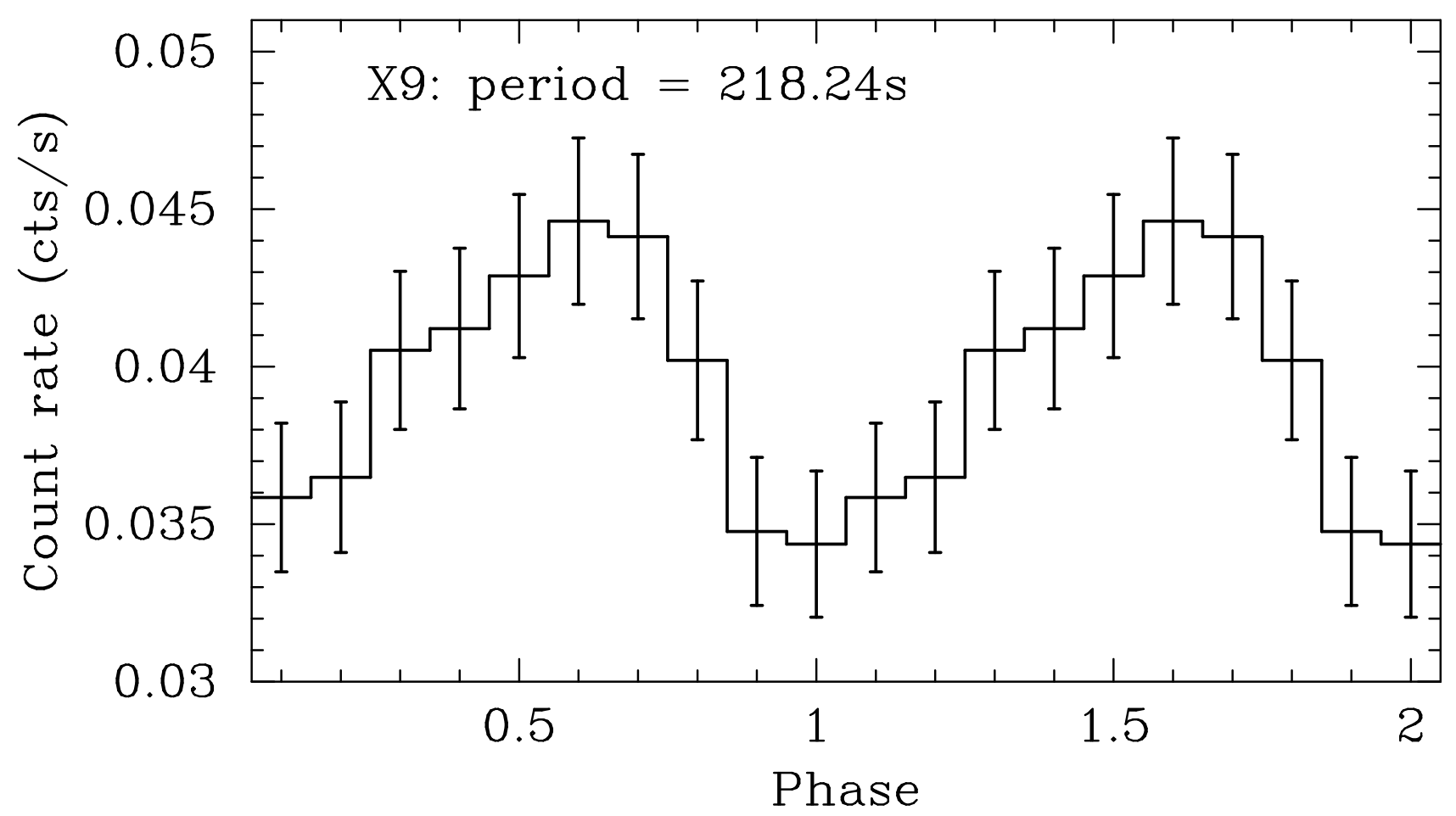

Fig. 4. Folded light curve for the brightest CV candidate, X9, showing its probable detection as a 218.24s pulsed source and thus identification as an accreting magnetic WD, or magnetic CV system (a NS x-ray pulsar with such a long period would be expected only in a wind-fed high mass x-ray binary found only near regions of massive star formation in the Galaxy, not in globular clusters). 


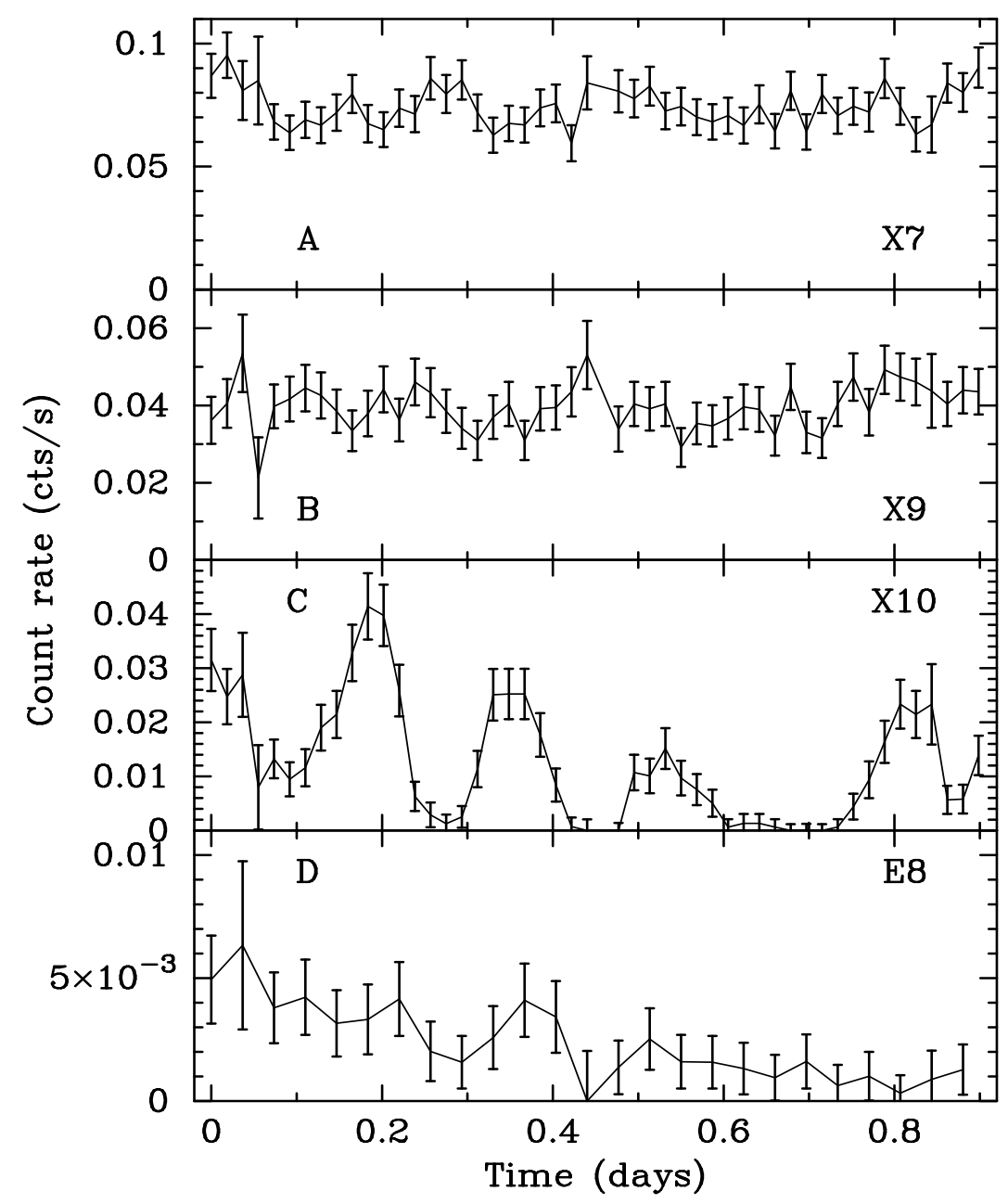

Fig. 5. Light curves, over the full $74 \mathrm{ks}$ (duration) observation, for 3 of the 4 source types (all MSP fluxes are constant, within statistics). (A) quiescent LMXB source X7; (B) CV candidates X9 (cf. Fig. 4), showing flickering, and (C) X10, showing dips and eclipses with 3.8h period (similar to X5); and (D) M-S binary candidate E8, showing smooth decline from probable large flare. 


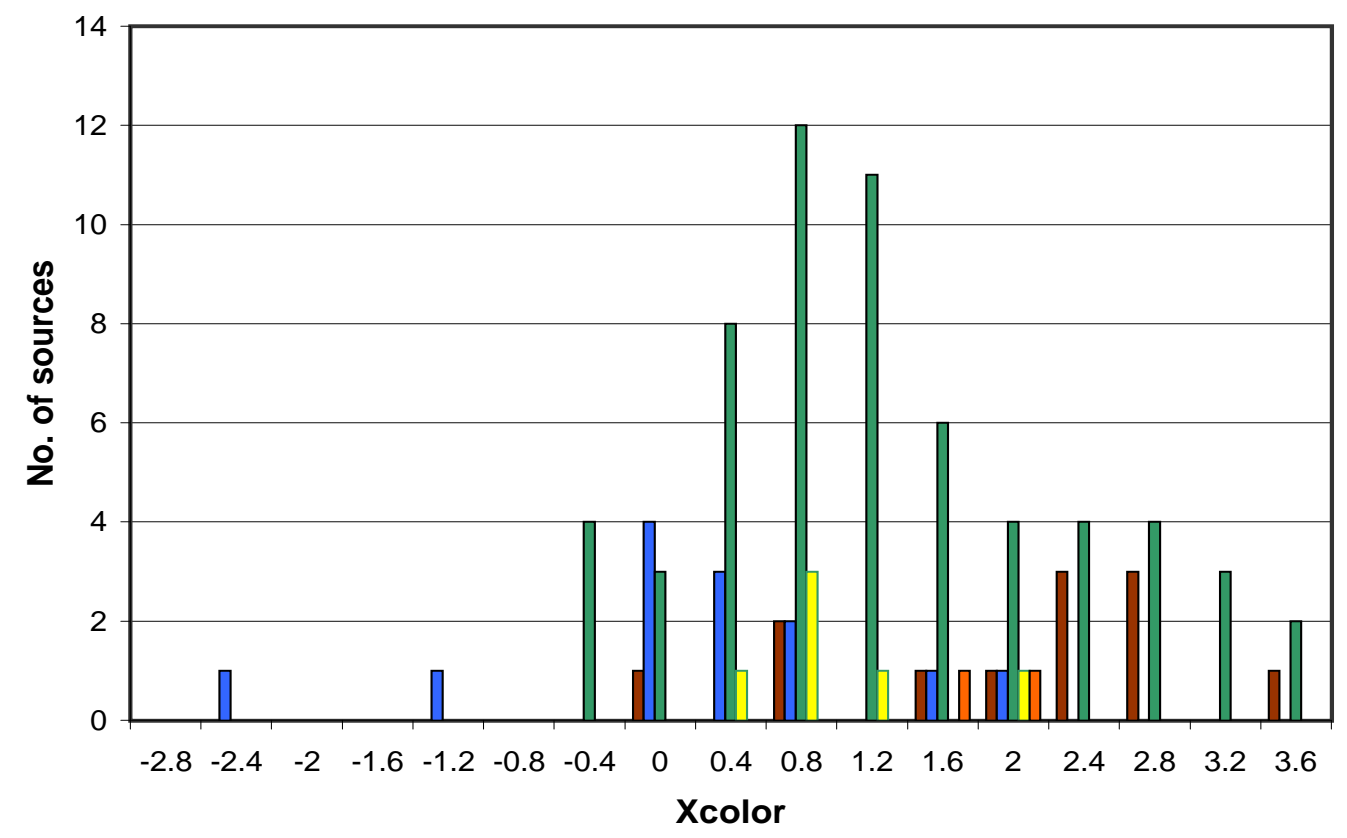

Fig. 6. Distributions of x-ray color (cf. Fig. 3) for 94 sources with medcts $\gtrsim 5$ counts and proposed source identifications (see Figs. 1 and 3; source types are color coded same as in Fig. 3). M-S binaries are BY Dra systems in flaring outbursts. Means and standard deviations for each source distribution are given in the text. 
Table 1. 47 Tuc Core X-Ray Sources (Grindlay et al 2001, Science On Line, 17 May 2001)

\begin{tabular}{|c|c|c|c|c|c|c|c|c|}
\hline $\operatorname{Src}^{a}$ & $\mathrm{RA}^{b}$ & $\operatorname{Dec}^{b}$ & Medcts $^{c}$ & Sfcts & Hdcts & $\mathrm{Sf} / \mathrm{Hd}^{d}$ & $L_{X}^{e}$ & $\mathrm{ID}^{f}$ \\
\hline W1 & $0: 24: 16.969 \pm 0.007$ & $-72: 04: 27.18 \pm 0.03$ & 229.7 & 117.4 & 124.6 & 0.9 & $4.8 \mathrm{e} 31$ & $\mathrm{CV}$ \\
\hline W2 & $0: 24: 15.89 \pm 0.007$ & $-72: 04: 36.37 \pm 0.03$ & 187.3 & 98.1 & 104.6 & 0.9 & $3.9 \mathrm{e} 31$ & $\mathrm{X} 13, \mathrm{CV}$ \\
\hline W3 & $0: 24: 15.163 \pm 0.02$ & $-72: 04: 43.36 \pm 0.09$ & 34.6 & 29.7 & 6.1 & 4.8 & $7.3 \mathrm{e} 30$ & - \\
\hline W4 & $0: 24: 13.464 \pm 0.01$ & $-72: 04: 51.13 \pm 0.08$ & 30.3 & 22.1 & 10.3 & 2.1 & $6.4 \mathrm{e} 30$ & - \\
\hline W5 & $0: 24: 12.58 \pm 0.02$ & $-72: 04: 41.10 \pm 0.05$ & 8.2 & 7.5 & 1 & 7.7 & $1.7 \mathrm{e} 30$ & - \\
\hline W6 & $0: 24: 11.649 \pm 0.03$ & $-72: 05: 03.69 \pm 0.1$ & 9.4 & 9.4 & 0.5 & 20 & $2.0 \mathrm{e} 30$ & - \\
\hline W7 & $0: 24: 11.125 \pm 0.02$ & $-72: 05: 20.14 \pm 0.06$ & 23.4 & 21.5 & 2 & 11 & $4.9 \mathrm{e} 30$ & MSP-E \\
\hline W8 & $0: 24: 10.763 \pm 0.01$ & $-72: 04: 25.71 \pm 0.05$ & 65.2 & 6.4 & 78.5 & 0.1 & $1.4 \mathrm{e} 31$ & $\mathrm{CV}$ \\
\hline W9 & $0: 24: 10.512 \pm 0.02$ & $-72: 05: 06.35 \pm 0.2$ & 9.6 & 5.7 & 4.3 & 1.3 & $2.0 \mathrm{e} 30$ & - \\
\hline W10 & $0: 24: 10.07 \pm 0.02$ & $-72: 04: 41.23 \pm 0.09$ & 18.5 & 13.2 & 5.7 & 2.3 & $3.9 \mathrm{e} 30$ & - \\
\hline W11 & 0:24:09.844 \pm 0.02 & $-72: 03: 59.69 \pm 0.1$ & 12.4 & 7.7 & 3.9 & 2 & $2.6 \mathrm{e} 30$ & MSP-U \\
\hline W12 & 0:24:09.514 \pm 0.02 & $-72: 05: 04.58 \pm 0.1$ & 8.3 & 6.6 & 2 & 3.3 & $1.7 \mathrm{e} 30$ & - \\
\hline W13 & 0:24:09.193 \pm 0.03 & $-72: 04: 28.83 \pm 0.1$ & 10.2 & 9.4 & 1 & 9.1 & $2.1 \mathrm{e} 30$ & MSP-N \\
\hline W14 & $0: 24: 08.78 \pm 0.02$ & $-72: 05: 07.46 \pm 0.08$ & 39.5 & 26.6 & 12.9 & 2.1 & $8.3 \mathrm{e} 30$ & M-S \\
\hline W15 & 0:24:08.496 \pm 0.01 & $-72: 05: 00.23 \pm 0.05$ & 78.8 & 18.5 & 69.9 & 0.3 & $1.7 \mathrm{e} 31$ & $\mathrm{CV}$ \\
\hline W16 & 0:24:08.299 \pm 0.01 & $-72: 04: 35.68 \pm 0.06$ & 93.7 & 55.3 & 42.6 & 1.3 & $2.0 \mathrm{e} 31$ & - \\
\hline W17 & 0:24:08.32 \pm 0.009 & $-72: 04: 31.39 \pm 0.04$ & 96.6 & 74.5 & 24.2 & 3.1 & $2.0 \mathrm{e} 31$ & - \\
\hline W18 & $0: 24: 08.17 \pm 0.02$ & $-72: 05: 13.29 \pm 0.09$ & 21.1 & 12.8 & 7.4 & 1.7 & $4.4 \mathrm{e} 30$ & M-S \\
\hline W19 & 0:24:07.97 \pm 0.02 & $-72: 04: 39.64 \pm 0.07$ & 17.1 & 15.1 & 2 & 7.7 & $3.6 \mathrm{e} 30$ & MSP-G,I \\
\hline W20 & 0:24:07.924 \pm 0.02 & $-72: 04: 54.75 \pm 0.05$ & 53.4 & 33.9 & 21.1 & 1.6 & $1.1 \mathrm{e} 31$ & - \\
\hline
\end{tabular}


Table 1-Continued

\begin{tabular}{|c|c|c|c|c|c|c|c|c|}
\hline $\operatorname{Src}^{a}$ & $\mathrm{RA}^{b}$ & $\operatorname{Dec}^{b}$ & Medcts $^{c}$ & Sfcts & Hdcts & $\mathrm{Sf} / \mathrm{Hd}^{d}$ & $L_{X}^{e}$ & $\mathrm{ID}^{f}$ \\
\hline W21 & 0:24:07.773 \pm 0.01 & $-72: 05: 27.10 \pm 0.05$ & 60.3 & 37.2 & 24.5 & 1.5 & $1.3 \mathrm{e} 31$ & $\mathrm{CV}$ \\
\hline W22 & 0:24:07.832 \pm 0.02 & $-72: 05: 24.38 \pm 0.09$ & 10.4 & 5.8 & 5 & 1.2 & $2.2 \mathrm{e} 30$ & - \\
\hline W23 & $0: 24: 07.804 \pm 0.009$ & $-72: 04: 41.57 \pm 0.05$ & 93.2 & 48.8 & 47.7 & 1 & $2.0 \mathrm{e} 31$ & - \\
\hline W24 & 0:24:07.376 \pm 0.02 & $-72: 04: 49.41 \pm 0.05$ & 41.5 & 40.1 & 12 & 3.3 & $8.7 \mathrm{e} 30$ & - \\
\hline W25 & $0: 24: 07.141 \pm 0.009$ & $-72: 05: 45.67 \pm 0.04$ & 134.7 & 86.5 & 53.3 & 1.6 & $2.8 \mathrm{e} 31$ & $\mathrm{X} 11, \mathrm{CV}$ \\
\hline W26 & 0:24:06.869 \pm 0.01 & $-72: 05: 12.29 \pm 0.08$ & 25.5 & 17.7 & 7.7 & 2.3 & $5.4 \mathrm{e} 30$ & - \\
\hline $\mathrm{W} 27$ & 0:24:06.397 \pm 0.003 & $-72: 04: 42.97 \pm 0.01$ & 802.4 & 626.5 & 201.7 & 3.1 & $1.7 \mathrm{e} 32$ & $\mathrm{X} 10, \mathrm{~V} 3, \mathrm{CV}$ \\
\hline W28 & $0: 24: 06.039 \pm 0.02$ & $-72: 05: 01.6 \pm 0.1$ & 16 & 15.9 & 1 & 16.7 & $3.4 \mathrm{e} 30$ & - \\
\hline W29 & 0:24:06.064 \pm 0.01 & $-72: 04: 48.93 \pm 0.07$ & 41.3 & 27.7 & 19 & 1.4 & $8.7 \mathrm{e} 30$ & - \\
\hline W30 & $0: 24: 06.013 \pm 0.005$ & $-72: 04: 56.20 \pm 0.03$ & 307.3 & 166.6 & 155.5 & 1.1 & $6.5 \mathrm{e} 31$ & $\mathrm{X} 19, \mathrm{~V} 2, \mathrm{CV}$ \\
\hline W31 & $0: 24: 05.662 \pm 0.03$ & $-72: 05: 04.51 \pm 0.08$ & 5.5 & 6 & 0.5 & 12 & $1.2 \mathrm{e} 30$ & - \\
\hline W32 & 0:24:05.656 \pm 0.01 & $-72: 04: 49.15 \pm 0.04$ & 76.8 & 50.6 & 27.3 & 1.9 & $1.6 \mathrm{e} 31$ & - \\
\hline W33 & 0:24:05.41 \pm 0.02 & $-72: 04: 21.36 \pm 0.1$ & 18.1 & 7.7 & 12.1 & 0.6 & $3.8 \mathrm{e} 30$ & - \\
\hline W34 & 0:24:05.216 \pm 0.01 & $-72: 04: 46.59 \pm 0.07$ & 30.2 & 22 & 10.6 & 2.1 & $6.3 \mathrm{e} 30$ & - \\
\hline W35 & $0: 24: 05.02 \pm 0.02$ & $-72: 05: 06.22 \pm 0.1$ & 23.5 & 13.9 & 10.2 & 1.4 & $4.9 \mathrm{e} 30$ & - \\
\hline W36 & 0:24:04.967 \pm 0.02 & $-72: 04: 55.4 \pm 0.08$ & 35.8 & 30.9 & 7 & 4.3 & $7.5 \mathrm{e} 30$ & $\mathrm{AKO} 9, \mathrm{CV}$ \\
\hline W37 & 0:24:05.007 \pm 0.02 & $-72: 04: 51.27 \pm 0.09$ & 35.2 & 21.8 & 14.5 & 1.5 & $7.4 \mathrm{e} 30$ & - \\
\hline W38 & $0: 24: 04.902 \pm 0.01$ & $-72: 04: 46.19 \pm 0.07$ & 14 & 9 & 6 & 1.5 & $2.9 \mathrm{e} 30$ & - \\
\hline W39 & 0:24:04.637 \pm 0.02 & $-72: 04: 53.71 \pm 0.1$ & 20.2 & 19.2 & 3 & 6.3 & $4.2 \mathrm{e} 30$ & MSP-O \\
\hline W40 & $0: 24: 04.62 \pm 0.02$ & $-72: 04: 34.1 \pm 0.2$ & 22.9 & 21.1 & 2 & 11.1 & $4.8 \mathrm{e} 30$ & - \\
\hline
\end{tabular}


Table 1-Continued

\begin{tabular}{|c|c|c|c|c|c|c|c|c|}
\hline $\mathrm{Src}^{a}$ & $\mathrm{RA}^{b}$ & $\operatorname{Dec}^{b}$ & Medcts $^{c}$ & Sfcts & Hdcts & $\mathrm{Sf} / \mathrm{Hd}^{d}$ & $L_{X}{ }^{e}$ & $\mathrm{ID}^{f}$ \\
\hline W41 & $0: 24: 04.345 \pm 0.02$ & $-72: 05: 01.35 \pm 0.08$ & 27.6 & 20.7 & 9 & 2.3 & $5.8 \mathrm{e} 30$ & M-S \\
\hline $\mathrm{W} 42$ & $0: 24: 04.263 \pm 0.002$ & $-72: 04: 57.97 \pm 0.01$ & 2402 & 1451.4 & 1154.8 & 1.3 & $5 \mathrm{e} 32$ & $\mathrm{X} 9, \mathrm{~V} 1, \mathrm{CV}$ \\
\hline W43 & $0: 24: 04.276 \pm 0.01$ & $-72: 04: 43.52 \pm 0.1$ & 21.9 & 11 & 13.1 & 0.8 & $4.6 \mathrm{e} 30$ & M-S \\
\hline W44 & $0: 24: 03.703 \pm 0.01$ & $-72: 04: 59.03 \pm 0.05$ & 24.5 & 18 & 23 & 0.8 & $5.1 \mathrm{e} 30$ & $\mathrm{CV}$ \\
\hline $\mathrm{W} 45$ & $0: 24: 03.776 \pm 0.006$ & $-72: 04: 22.96 \pm 0.02$ & 250.6 & 134.1 & 128.9 & 1 & $5.3 \mathrm{e} 31$ & $\mathrm{CV}$ \\
\hline $\mathrm{W} 46$ & $0: 24: 03.515 \pm 0.001$ & $-72: 04: 51.91 \pm 0.01$ & 5332.2 & 4329.5 & 989.7 & 4.3 & $1.1 \mathrm{e} 33$ & X7,qLMXB \\
\hline $\mathrm{W} 47$ & $0: 24: 03.464 \pm 0.008$ & $-72: 05: 05.20 \pm 0.03$ & 157 & 88.5 & 72.3 & 1.2 & $3.3 \mathrm{e} 31$ & E8,M-S \\
\hline $\mathrm{W} 48$ & $0: 24: 03.217 \pm 0.02$ & $-72: 04: 29.62 \pm 0.1$ & 15.7 & 12.4 & 4 & 3.1 & $3.3 \mathrm{e} 30$ & - \\
\hline W49 & $0: 24: 03.083 \pm 0.02$ & $-72: 04: 47.28 \pm 0.07$ & 12.8 & 5 & 9 & 0.6 & $2.7 \mathrm{e} 30$ & - \\
\hline W50 & $0: 24: 03.14 \pm 0.02$ & $-72: 04: 26.54 \pm 0.08$ & 16.6 & 13.5 & 4 & 3.3 & $3.5 \mathrm{e} 30$ & - \\
\hline W51 & $0: 24: 02.806 \pm 0.01$ & $-72: 04: 49.04 \pm 0.06$ & 46.3 & 33.9 & 13 & 2.6 & $9.7 \mathrm{e} 30$ & - \\
\hline W52 & $0: 24: 02.597 \pm 0.02$ & $-72: 05: 38.41 \pm 0.1$ & 11.8 & 7.9 & 3 & 2.6 & $2.5 \mathrm{e} 30$ & - \\
\hline W53 & $0: 24: 02.533 \pm 0.01$ & $-72: 05: 11.25 \pm 0.06$ & 60.6 & 32.9 & 32.3 & 1 & $1.3 \mathrm{e} 31$ & - \\
\hline W54 & $0: 24: 02.522 \pm 0.02$ & $-72: 04: 44.24 \pm 0.09$ & 20.9 & 15.7 & 5.3 & 2.9 & $4.4 \mathrm{e} 30$ & - \\
\hline W55 & $0: 24: 02.226 \pm 0.03$ & $-72: 04: 50.63 \pm 0.1$ & 9.9 & 6.9 & 4 & 1.7 & $2.1 \mathrm{e} 30$ & - \\
\hline W56 & $0: 24: 02.141 \pm 0.004$ & $-72: 05: 41.94 \pm 0.02$ & 695 & 313.8 & 412.9 & 0.8 & $1.5 \mathrm{e} 32$ & $\mathrm{X} 6, \mathrm{CV}$ \\
\hline W57 & $0: 24: 01.37 \pm 0.03$ & $-72: 04: 45.55 \pm 0.07$ & 10.1 & 6 & 4.7 & 1.3 & $2.1 \mathrm{e} 30$ & - \\
\hline W58 & $0: 24: 00.968 \pm 0.001$ & $-72: 04: 53.18 \pm 0.01$ & 4435.2 & 3455.7 & 981.4 & 3.6 & $9.2 \mathrm{e} 32$ & X5,qLMXB \\
\hline W59 & 0:24:00.627士0.02 & $-72: 05: 54.13 \pm 0.1$ & 18 & 9.1 & 9.9 & 0.9 & $3.8 \mathrm{e} 30$ & - \\
\hline W60 & 0:24:00.043 \pm 0.02 & $-72: 05: 12.85 \pm 0.09$ & 5.4 & 4.6 & 1 & 4.5 & $1.1 \mathrm{e} 30$ & - \\
\hline
\end{tabular}


Table 1-Continued

\begin{tabular}{|c|c|c|c|c|c|c|c|c|}
\hline $\mathrm{Src}^{a}$ & $\mathrm{RA}^{b}$ & $\operatorname{Dec}^{b}$ & Medcts $^{c}$ & Sfcts & Hdcts & $\mathrm{Sf} / \mathrm{Hd}^{d}$ & $L_{X}^{e}$ & $\mathrm{ID}^{f}$ \\
\hline W61 & $0: 23: 59.877 \pm 0.02$ & $-72: 05: 04.65 \pm 0.1$ & 20.9 & 14.8 & 5 & 2.9 & $4.4 \mathrm{e} 30$ & - \\
\hline W62 & $0: 23: 59.434 \pm 0.02$ & $-72: 04: 47.79 \pm 0.2$ & 9.5 & 7.9 & 2 & 4 & $2.0 \mathrm{e} 30$ & - \\
\hline W63 & $0: 23: 59.392 \pm 0.03$ & $-72: 03: 58.81 \pm 0.08$ & 10 & 5.9 & 5.9 & 1 & $3.0 \mathrm{e} 30$ & MSP-J \\
\hline W64 & 0:23:57.699 \pm 0.007 & $-72: 05: 01.93 \pm 0.03$ & 164.2 & 88.4 & 87.4 & 1 & $3.4 \mathrm{e} 31$ & - \\
\hline W65 & $0: 23: 53.548 \pm 0.02$ & $-72: 05: 11.20 \pm 0.1$ & 9.7 & 6.8 & 3 & 2.3 & $4.0 \mathrm{e} 30$ & - \\
\hline W66 & $0: 24: 16.023 \pm 0.03$ & $-72: 04: 51.56 \pm 0.2$ & 10.3 & 10.3 & 0.5 & 20 & $2.2 \mathrm{e} 30$ & - \\
\hline W67 & $0: 24: 13.818 \pm 0.03$ & $-72: 04: 43.83 \pm 0.1$ & 13.9 & 8.9 & 5.6 & 1.6 & $2.9 \mathrm{e} 30$ & MSP-D \\
\hline W68 & $0: 24: 13.477 \pm 0.02$ & $-72: 05: 23.42 \pm 0.2$ & 6.4 & 5.4 & 1 & 5.3 & $1.3 \mathrm{e} 30$ & M-S \\
\hline W69 & $0: 24: 12.643 \pm 0.02$ & $-72: 04: 22.38 \pm 0.2$ & 3.9 & 2.8 & 2 & 1.4 & $8.2 \mathrm{e} 29$ & - \\
\hline W70 & $0: 24: 11.915 \pm 0.02$ & $-72: 04: 43.84 \pm 0.1$ & 11 & 8 & 3 & 2.6 & $2.3 \mathrm{e} 30$ & - \\
\hline W71 & $0: 24: 10.642 \pm 0.01$ & $-72: 05: 16.37 \pm 0.2$ & 8.6 & 6.9 & 1 & 7.1 & $1.8 \mathrm{e} 30$ & - \\
\hline W72 & $0: 24: 10.484 \pm 0.02$ & $-72: 04: 59.92 \pm 0.1$ & 16.6 & 15.1 & 3 & 5 & $3.5 \mathrm{e} 30$ & - \\
\hline W73 & $0: 24: 07.224 \pm 0.02$ & $-72: 04: 57.39 \pm 0.1$ & 13.5 & 8.1 & 2 & 4 & $2.8 \mathrm{e} 30$ & - \\
\hline W74 & $0: 24: 06.686 \pm 0.02$ & $-72: 04: 06.74 \pm 0.1$ & 10.4 & 10.6 & 0.5 & 21 & $2.2 \mathrm{e} 30$ & MSP-H \\
\hline W75 & $0: 24: 06.429 \pm 0.02$ & $-72: 04: 52.79 \pm 0.1$ & 24.6 & 15 & 8 & 2 & $5.2 \mathrm{e} 30$ & - \\
\hline W76 & $0: 24: 6.436 \pm 0.04$ & $-72: 04: 30.11 \pm 0.1$ & 5.1 & 2 & 4 & 0.5 & $1.1 \mathrm{e} 30$ & - \\
\hline W77 & $0: 24: 03.92 \pm 0.02$ & $-72: 04: 42.71 \pm 0.08$ & 27.3 & 19.8 & 6 & 3.3 & $5.7 \mathrm{e} 30$ & MSP-F,S \\
\hline W78 & $0: 24: 03.516 \pm 0.02$ & $-72: 05: 22.54 \pm 0.1$ & 4.2 & 4.3 & 0.5 & 8.6 & $8.8 \mathrm{e} 29$ & - \\
\hline W79 & $0: 24: 03.565 \pm 0.02$ & $-72: 04: 41.50 \pm 0.1$ & 19.1 & 14 & 8.6 & 1.6 & $4.0 \mathrm{e} 30$ & - \\
\hline W80 & $0: 24: 02.658 \pm 0.02$ & $-72: 04: 41.47 \pm 0.1$ & 11.4 & 8.5 & 2 & 4.2 & $2.4 \mathrm{e} 30$ & - \\
\hline
\end{tabular}


Table 1-Continued

\begin{tabular}{|c|c|c|c|c|c|c|c|c|}
\hline $\operatorname{Src}^{a}$ & $\mathrm{RA}^{b}$ & $\operatorname{Dec}^{b}$ & Medcts $^{c}$ & Sfcts & Hdcts & $\mathrm{Sf} / \mathrm{Hd}^{d}$ & $L_{X}^{e}$ & $\operatorname{ID}^{f}$ \\
\hline W81 & $0: 24: 01.775 \pm 0.03$ & $-72: 05: 12.30 \pm 0.1$ & 7.9 & 4 & 5.2 & 0.8 & $1.7 \mathrm{e} 30$ & - \\
\hline W82 & $0: 24: 01.434 \pm 0.02$ & $-72: 04: 41.72 \pm 0.1$ & 8.6 & 8.9 & 0.5 & 18 & $1.8 \mathrm{e} 30$ & - \\
\hline W83 & $0: 24: 00.977 \pm 0.03$ & $-72: 04: 13.70 \pm 0.1$ & 9.5 & 7.6 & 3 & 2.6 & $2.0 \mathrm{e} 30$ & - \\
\hline W84 & $0: 24: 00.424 \pm 0.04$ & $-72: 05: 46.55 \pm 0.2$ & 6 & 2 & 3 & 0.7 & $1.3 \mathrm{e} 30$ & - \\
\hline W85 & $0: 23: 59.404 \pm 0.04$ & $-72: 04: 38.46 \pm 0.1$ & 9.5 & 8.5 & 1 & 8.3 & $2.0 \mathrm{e} 30$ & - \\
\hline W86 & $0: 23: 58.988 \pm 0.02$ & $-72: 05: 09.70 \pm 0.1$ & 3.8 & 3 & 1 & 3 & $8 \mathrm{e} 29$ & - \\
\hline W87 & $0: 23: 57.884 \pm 0.04$ & $-72: 04: 52.76 \pm 0.08$ & 7.4 & 4.5 & 3 & 1.5 & $1.6 \mathrm{e} 30$ & - \\
\hline W88 & $0: 23: 54.175 \pm 0.03$ & $-72: 05: 14.50 \pm 0.1$ & 4.6 & 3 & 2 & 1.5 & $1.7 \mathrm{e} 30$ & - \\
\hline W89 & $0: 23: 52.544 \pm 0.03$ & $-72: 05: 32.99 \pm 0.1$ & 3 & 3 & 0.5 & 6 & $1.6 \mathrm{e} 30$ & - \\
\hline W90 & $0: 23: 50.806 \pm 0.03$ & $-72: 05: 41.51 \pm 0.1$ & 8.3 & 5.4 & 3 & 1.8 & $2.1 \mathrm{e} 30$ & - \\
\hline W91 & $0: 24: 17.55 \pm 0.05$ & $-72: 03: 58.28 \pm 0.1$ & 3.7 & 0.5 & 4 & 0.13 & $7.8 \mathrm{e} 29$ & - \\
\hline W92 & $0: 24: 15.678 \pm 0.02$ & $-72: 04: 41.31 \pm 0.2$ & 9.2 & 7 & 3 & 2.3 & $1.9 \mathrm{e} 30$ & - \\
\hline W93 & $0: 24: 12.017 \pm 0.02$ & $-72: 05: 08.06 \pm 0.2$ & 3.8 & 2 & 2 & 1 & $8.0 \mathrm{e} 29$ & - \\
\hline W94 & $0: 24: 11.78 \pm 0.03$ & $-72: 05: 14.61 \pm 0.09$ & 5.5 & 4 & 2 & 2 & $1.2 \mathrm{e} 30$ & - \\
\hline W95 & $0: 24: 10.861 \pm 0$ & $-72: 04: 15.84 \pm 0.1$ & 2.9 & 3 & 0.5 & 6 & $6.1 \mathrm{e} 29$ & - \\
\hline W96 & $0: 24: 07.281 \pm 0.02$ & $-72: 05: 19.58 \pm 0.2$ & 4.4 & 4 & 1 & 4 & $9.2 \mathrm{e} 29$ & - \\
\hline W97 & $0: 24: 05.379 \pm 0.02$ & $-72: 05: 11.12 \pm 0.1$ & 5.5 & 5 & 1 & 5 & $1.2 \mathrm{e} 30$ & - \\
\hline W98 & $0: 24: 05.056 \pm 0.03$ & $-72: 05: 02.77 \pm 0.1$ & 10.5 & 11 & 0.5 & 22 & $2.2 \mathrm{e} 30$ & - \\
\hline W99 & $0: 24: 02.37 \pm 0.04$ & $-72: 05: 50.66 \pm 0.09$ & 4.7 & 4 & 2 & 2 & $9.9 \mathrm{e} 29$ & - \\
\hline W100 & $0: 24: 01.368 \pm 0.02$ & $-72: 04: 27.26 \pm 0.2$ & 5.4 & 5 & 0.5 & 10 & $1.1 \mathrm{e} 30$ & - \\
\hline
\end{tabular}


Table 1 - Continued

\begin{tabular}{|c|c|c|c|c|c|c|c|c|}
\hline $\mathrm{Src}^{a}$ & $\mathrm{RA}^{b}$ & $\operatorname{Dec}^{b}$ & Medcts $^{c}$ & Sfcts & Hdcts & $\mathrm{Sf} / \mathrm{Hd}^{d}$ & $L_{X}^{e}$ & $\operatorname{ID}^{f}$ \\
\hline W101 & $0: 23: 59.974 \pm 0.02$ & $-72: 05: 31.93 \pm 0.2$ & 5.4 & 5 & 0.5 & 10 & $1.1 \mathrm{e} 30$ & - \\
\hline W102 & $0: 23: 57.687 \pm 0.05$ & $-72: 05: 42.98 \pm 0.1$ & 2.8 & 3 & 0.5 & 6 & $5.9 \mathrm{e} 29$ & - \\
\hline W103 & $0: 23: 51.996 \pm 0.04$ & $-72: 04: 29.31 \pm 0.1$ & 1.9 & 2 & 0.5 & 4 & $4.0 \mathrm{e} 29$ & - \\
\hline W104 & $0: 24: 16.49$ & $-72: 04: 25.15$ & 8 & 7 & 1 & 7 & $1.7 \mathrm{e} 30$ & MSP-Q \\
\hline W105 & $0: 24: 08.54$ & $-72: 04: 38.91$ & 6 & 5 & 1 & 5 & $1.3 \mathrm{e} 30$ & MSP-T \\
\hline W106 & 0:24:03.77 & $-72: 04: 56.90$ & 12 & 11 & 1 & 11 & $2.5 \mathrm{e} 30$ & MSP-L \\
\hline W107 & $0: 23: 54.49$ & $-72: 05: 30.72$ & 3 & 3 & 0.5 & 6 & $2.3 \mathrm{e} 30$ & MSP-M \\
\hline W108 & $0: 23: 50.35$ & $-72: 04: 31.49$ & 1 & 1 & 0.5 & 2 & $1.3 \mathrm{e} 30$ & MSP-C \\
\hline
\end{tabular}

Note. - ${ }^{a}$ Chandra source number. ${ }^{b}$ RA (right ascension) and Dec (declination) coordinates are given as epoch J2000. Errors are formal $1 \sigma$ centroiding errors from Chandra x-ray detection routine wavdetect. The final five MSPs were not formally detected by wavdetect, so the radio positions of Freire et al, 2001 (12) are given without errors. ${ }^{c}$ Medcts=counts in $0.5-4.5 \mathrm{keV}$ band, Sfcts=counts in $0.5-1.5 \mathrm{keV}$ band, Hdcts=counts in $1.5-6 \mathrm{keV}$ band. Values of 0.5 counts are estimated upper limits. ${ }^{d}$ Softness ratio, defined as Sfcts/Hdcts [in Figs. 3 and 6, Xcolor $=2.5 \log$ (Sfcts/Hdcts)]. ${ }^{e}$ Intrinsic luminosities in ROSAT (0.5-2.5 keV) band, with multiplicative exposure corrections (=1.4, 2.0, 1.7, 2.6, 1.2, 2.5 and 1.9 for sources W63(MSPJ), W65, W88, W89, W90, MSPC and MSPM, respectively), based on 1 keV thermal bremsstrahlung spectrum, $N_{H}=2.4 \times 10^{20} \mathrm{~cm}^{-} 2$, d=5.0 kpc. ${ }^{f} \mathrm{X}$ number from ROSAT (14) observations (e.g. X5), certain optical or radio counterparts (e.g. V1, AKO9, MSP-D), and class identifications: $\mathrm{qLMXB}=$ quiescent $\mathrm{LMXB}, \mathrm{M}-\mathrm{S}=$ main sequence binary, $\mathrm{CV}=$ cataclysmic variable. Detailed discussion of of individual x-ray source spectra and variability is deferred to Heinke et al. 2001 (21) and probable optical counterparts are discussed by Edmonds et al. 2001 (22). 